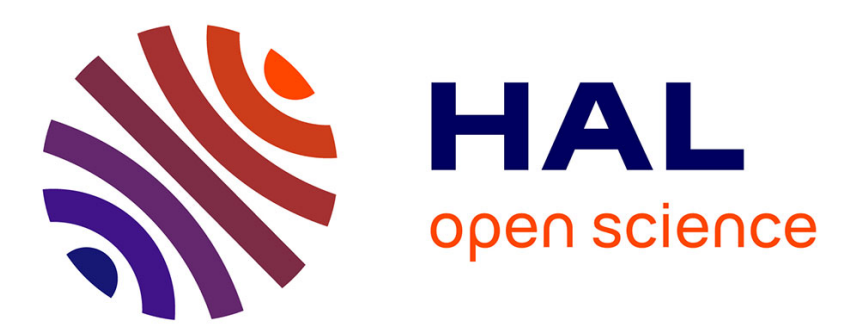

\title{
Identification of the strain rate parameters for structural adhesives
}

Eduardo Fancello, Luca Goglio, Laurent Stainier, Jakson Manfredini Vassoler

\section{To cite this version:}

Eduardo Fancello, Luca Goglio, Laurent Stainier, Jakson Manfredini Vassoler. Identification of the strain rate parameters for structural adhesives. Journal of Adhesion Science and Technology, 2008, 22, pp.1523-1540. 10.1163/156856108X309495 . hal-01006950

\section{HAL Id: hal-01006950 \\ https://hal.science/hal-01006950}

Submitted on 15 Nov 2021

HAL is a multi-disciplinary open access archive for the deposit and dissemination of scientific research documents, whether they are published or not. The documents may come from teaching and research institutions in France or abroad, or from public or private research centers.
L'archive ouverte pluridisciplinaire HAL, est destinée au dépôt et à la diffusion de documents scientifiques de niveau recherche, publiés ou non, émanant des établissements d'enseignement et de recherche français ou étrangers, des laboratoires publics ou privés.

\section{다(1) $\$$}

Distributed under a Creative Commons Attribution - NonCommercial| 4.0 International 


\title{
Identification of the Strain Rate Parameters for Structural Adhesives
}

\author{
E. Fancello ${ }^{a}$, L. Goglio ${ }^{b, *}$, L. Stainier ${ }^{c}$ and J. M. Vassoler ${ }^{b}$ \\ a Departamento de Engenharia Mecânica, Universidade Federal de Santa Catarina Campus \\ Universitário, Trindade 88040-900, Florianópolis, SC, Brazil \\ b Dipartimento di Meccanica, Politecnico di Torino Corso Duca degli Abruzzi 24, 10129 Torino, Italy \\ ${ }^{\mathrm{c}}$ Dépt. Aérospatiale \& Mécanique (LTAS-MCT), Université de Liège Chemin des chevreuils 1, \\ B-4000 Liège, Belgium
}

\begin{abstract}
Structural adhesives are frequently used for applications (mostly in the automotive field) in which they are exposed to dynamic loads at high strain rates, that can cause plastic straining. Thus, such plastic behavior must be included and correctly reproduced by finite element simulations, for instance, to predict the worthiness of the structure against crash conditions.

This work makes use of a recently developed general framework for non-linear constitutive models, based on a variational formulation in which, at every load increment, the updates satisfy a minimum principle. The problem is faced in general terms, operating on potentials from which the constitutive equations are obtained; thus different behaviors can be treated by the specific models which are implemented in the potentials. This paper describes the application of this approach to the case of a structural bi-component epoxy adhesive, with the aim of describing its behavior under compression at different test velocities. A widely available data set on compression tests (at strain rate up to $10^{3} \mathrm{~s}^{-1}$ ) has been utilized to identify the parameters of the potentials and to evaluate the capability of the models to reproduce the measurements.
\end{abstract}

Keywords

Adhesives, elasto-viscoplasticity, strain rate

\section{Introduction}

In many applications of the adhesives, e.g. in the automotive and transportation fields, impact conditions are common or, however, they must be considered for design. Relevant applications are in the production of structural members for which the traditional joining techniques (welding, riveting) are replaced by adhesive bonding. In such cases, the adhesive itself must not only ensure a strength level no lower than that offered by the traditional techniques, but it can also be required to act as

\footnotetext{
${ }^{*}$ To whom correspondence should be addressed. Tel.: +39 011 5646934; Fax: +39 011 5646999; e-mail: luca.goglio@polito.it
} 
an additional medium for dissipating energy. An example of this is represented by the structural foams, recently available on the market, used to fill car body beams.

The virtual validation of the structure against crash conditions is carried out by Finite Element Method (FEM) simulations, in which the behavior of new materials such as adhesives must be correctly reproduced by means of models which must include their typical features, i.e. non-linear elastic and viscous behavior, and moreover the strain rate dependence should be accounted for. Clearly, in addition to their mathematical development, the models must be supported by experiments that should, on the one hand, allow for assessing the quantitative values of the related parameters, and, on the other hand, validate their capability to reproduce the reality. About this aspect, it must be remarked that, unlike the case of metals and other structural materials (for which many results are available), not many studies have been published regarding the strain rate dependence of adhesives under impact.

Regarding the simulation of the (non-linear) material behavior, considerable advance has been made in the last decade by the development of models under a variational formulation. The main advantages of such approaches are that: (i) they can be regarded under a unifying minimum principle, (ii) they are very flexible in the sense that they can accommodate a wide collection of material behaviors by varying the potentials assumed and (iii) they are suitable for error estimation. However, their application is not so widespread yet; thus there is a considerable interest in testing their capability to model practical cases. At the same time, there is a practical need to show how the material parameters appearing in the adopted formulae (potentials, etc.) can be identified from an experimental data base.

This paper is structured as follows. First, the theoretical formulation is briefly described in terms of mathematical quantities and principles involved (this is done also with the aim to give an essential outline to the reader not familiar with the variational formulation; clearly, the specialized literature must be consulted for a deeper insight). Second, the experimental tests and results are described. Then the choice of the models and the identification of the parameters are presented and the final conclusions are drawn.

\section{Symbols}

\section{Main Quantities}

$c_{j}$ ( $j$ th) eigenvalue

C right Cauchy-Green strain tensor

D rate of deformation tensor

F deformation gradient tensor

$J$ Jacobian of $\mathbf{F}$

L spatial gradient of the velocity tensor 
$m$ material constant (viscous potential)

$\mathbf{m}_{j}$ ( $j$ th) eigenvector

$\mathbf{M}_{j}$ ( $j$ th) eigenprojection matrix

$N^{\mathrm{e}}, N^{\mathrm{p}}$ number of parameters (elastic, plastic)

P first Piola-Kirchhoff stress tensor

$\mathbf{R}$ rotation matrix

$q$ accumulated plastic deformation

Q internal state variable

$t$ time

$\mathbf{X}$ reference configuration (undeformed)

$\mathbf{x}$ present configuration (deformed)

$W$ energy potential

$\alpha_{k}^{\mathrm{e}}$ material constants (elastic potential)

$\alpha, \alpha_{k}^{\mathrm{p}}$ material constants (plastic potential)

$\beta_{1}, \beta_{2}$ Lagrange multipliers

$\varepsilon_{j}^{\mathrm{e}}(j$ th) elastic logarithmic strain

$\lambda$ stretch

$\mu^{\mathrm{e}}, \mu_{k}^{\mathrm{e}}$ material constants (elastic potential)

$\mu, \mu_{k}^{\mathrm{p}}$ material constants (plastic potential)

$\Sigma_{0}$ yield stress

$\sigma$ Cauchy stress tensor

$\phi, \psi$ dissipative potentials

$\Psi$ incremental potential (to be minimized)

Subscripts, Superscripts, Indices and Operators

e elastic

i inelastic

$\mathrm{p}$ plastic

$\mathrm{v}$ viscous

$j$ coordinate index 
$n$ time step index

-1 inverse (matrix or tensor)

$\mathrm{T}$ transpose (matrix or tensor)

isochoric

- time derivative (rate)

$\Delta$ increment

$\otimes$ dyadic product

: inner product

\section{Mathematical Background}

\subsection{Principles of the Continuum Mechanics Formulation}

To account for the non-linear behavior, the formulation is based on the typical quantities of continuum mechanics. First, the conservative aspect of the phenomenon is considered by assuming an energy potential [1], in the form $W=W\left(\mathbf{F}, \mathbf{F}^{\mathrm{i}}, \mathbf{Q}\right)$. In $W, \mathbf{F}=\partial \mathbf{x} / \partial \mathbf{X}$ accounts for the total deformation, whilst $\mathbf{F}^{\mathrm{i}}$ represents the inelastic deformation only; they are related by the multiplicative decomposition $\mathbf{F}=\mathbf{F}^{\mathrm{e}} \mathbf{F}^{\mathrm{i}}$ in which the elastic part $\mathbf{F}^{\mathrm{e}}$ also appears [2]. $\mathbf{Q}$ contains other internal variables to account for hardening. The whole set of internal variables formally includes both $\mathbf{F}^{\mathrm{i}}$ and $\mathbf{Q}$, a priori considered as independent. Later, in the process of adapting the formulation to specific constitutive behaviors (viscoplasticity), we will introduce relations linking $\mathbf{F}^{\mathrm{i}}$ and $\mathbf{Q}$ (e.g. a flow rule in plasticity - Section 2.2.2). These relations should be seen as non-holonomic constraints between $\mathbf{F}^{\mathrm{i}}$ and $\mathbf{Q}$, which have to be taken into account in the minimization process, and which in practice render $\mathbf{F}^{\mathrm{i}}$ dependent on the subset of internal variables $\mathbf{Q}$. Thus, for the sake of clarity, we make the distinction between $\mathbf{F}^{\mathrm{i}}$ and $\mathbf{Q}$ from the onset. By taking the time derivative of $W$, which gives its rate of change, one obtains:

$$
\dot{W}=\frac{\partial W}{\partial \mathbf{F}}: \dot{\mathbf{F}}+\frac{\partial W}{\partial \mathbf{F}^{\mathrm{i}}}: \dot{\mathbf{F}}^{\mathrm{i}}+\frac{\partial W}{\partial \mathbf{Q}}: \dot{\mathbf{Q}} .
$$

Second, the non-conservative aspects are considered by means of two pseudopotentials $\phi=\phi(\dot{\mathbf{F}})$, that accounts for the dependence of the material behavior on the total rate of deformation, and $\psi=\psi\left(\dot{\mathbf{F}}^{\mathrm{i}}, \dot{\mathbf{Q}}\right)$, that accounts for the dependence on the rate of inelastic deformation as well as on the rate of the internal variables. It can be proven [3] that the following relationships hold:

$$
\begin{gathered}
\mathbf{P}=\frac{\partial W}{\partial \mathbf{F}}+\frac{\partial \phi}{\partial \dot{\mathbf{F}}} \\
\frac{\partial W}{\partial \mathbf{F}^{\mathrm{i}}}+\frac{\partial \psi}{\partial \dot{\mathbf{F}}^{\mathrm{i}}}=0, \quad \frac{\partial W}{\partial \mathbf{Q}}+\frac{\partial \psi}{\partial \dot{\mathbf{Q}}}=0 .
\end{gathered}
$$


Equation (2) shows how the first Piola-Kirchhoff tensor can be expressed from appropriate potentials and equation $(3 a, b)$ define the restrictions on the evolution of the internal variables (originated from the self-equilibrium of the stresses conjugated to $\mathbf{F}^{\mathrm{i}}$ and $\mathbf{Q}$ ).

On the basis of the previous mathematical properties, the essence of the variational formulation is the definition of the potential $\Psi$, based on the work performed by the Piola-Kirchhoff stress (which is energetically conjugated to the rate of the deformation gradient) in the time interval $\left(0, t_{f}\right)$ :

$$
\Psi=\int_{0}^{t_{f}} \mathbf{P}: \dot{\mathbf{F}} \mathrm{d} t .
$$

In case of (hyper-)elastic material, the value of $\Psi$ would depend on the final state only. Due to the inelastic behavior, $\Psi$ depends also on the path followed during the evolution of the system. By minimizing the work, after several mathematical manipulations, it can be found that [4]:

$$
\delta \Psi=\mathbf{P}\left(t_{f}\right): \delta \mathbf{F}\left(t_{f}\right) .
$$

This allows for obtaining an expression for $\mathbf{P}$ :

$$
\mathbf{P}\left(t_{f}\right)=\frac{\partial \Psi\left(\mathbf{F}\left(t_{f}\right) ; \mathbf{F}(0), \mathbf{F}^{\mathrm{i}}(0), \mathbf{Q}(0)\right)}{\partial \mathbf{F}\left(t_{f}\right)},
$$

in which it is worth noting the dependence on all variables in the initial state $(t=0)$, and on $\mathbf{F}$ only in the final state $\left(t=t_{f}\right)$. In incremental terms, from the state $n$ to the state $n+1$, equation (6) becomes:

$$
\mathbf{P}_{n+1}=\frac{\partial \Psi\left(\mathbf{F}_{n+1} ; \mathbf{F}_{n}, \mathbf{F}_{n}^{\mathrm{i}}, \mathbf{Q}_{n}\right)}{\partial \mathbf{F}_{n+1}} .
$$

The incremental potential $\Psi\left(\mathbf{F}_{n+1} ; \mathbf{F}_{n}, \mathbf{F}_{n}^{\mathrm{i}}, \mathbf{Q}_{n}\right)$ can be written in the form [3]:

$$
\begin{aligned}
& \Psi\left(\mathbf{F}_{n+1} ; \mathbf{F}_{n}, \mathbf{F}_{n}^{\mathrm{i}}, \mathbf{Q}_{n}\right) \\
& \quad=\Delta t \phi(\stackrel{\circ}{\mathbf{F}})+\min _{\mathbf{F}_{n+1}^{\mathrm{i}}, \mathbf{Q}_{n+1}}\left\{W_{n+1}-W_{n}+\Delta t \psi\left(\stackrel{\circ}{\mathbf{F}}^{\mathrm{i}}, \stackrel{\circ}{\mathbf{Q}}\right)\right\},
\end{aligned}
$$

where $\stackrel{\circ}{\mathbf{F}}\left(\mathbf{F}_{n+1}\right), \stackrel{\circ}{\mathbf{F}}^{\mathrm{i}}\left(\mathbf{F}_{n+1}^{\mathrm{i}}\right)$ and $\stackrel{\circ}{\mathbf{Q}}\left(\mathbf{Q}_{n+1}\right)$ are, respectively, approximations of the rates $\dot{\mathbf{F}}, \dot{\mathbf{F}}^{\mathrm{i}}$ and $\dot{\mathbf{Q}}$. Equation (8) restates the constitutive equation (2) (from which $\mathbf{P}$ can be obtained) and, at the same time, satisfies the conditions (3) on the internal variables by means of the included minimization. The functions $W, \phi, \psi$ contained in (8) may assume different forms, according to the models adopted. The energy $W$ is, in general terms, additively expressed as:

$$
W=\varphi(\mathbf{F})+\varphi^{\mathrm{e}}\left(\mathbf{F}^{\mathrm{e}}\right)+\varphi^{\mathrm{i}}\left(\mathbf{F}^{\mathrm{i}}, \mathbf{Q}\right),
$$

where $\varphi, \varphi^{\mathrm{e}}, \varphi^{\mathrm{i}}$ are potentials related to the particular models adopted, as described in the following section. 


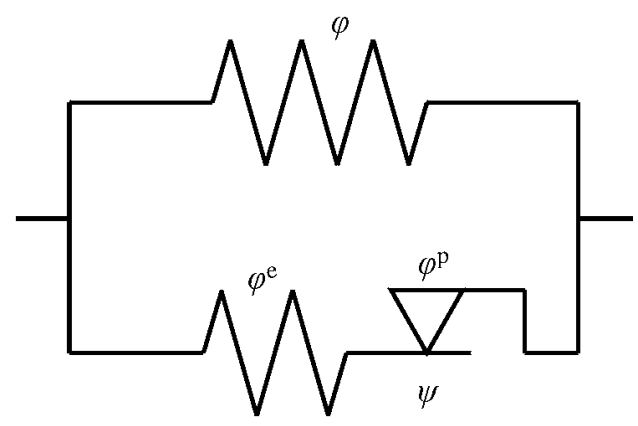

Figure 1. Rheological model for the elasto-viscoplastic behavior; $\varphi$ potential of the elastic branch, $\varphi^{\mathrm{e}}$ elastic potential of the Maxwell branch, $\varphi^{\mathrm{p}}$ plastic potential and $\psi$ viscoplastic potential.

\subsection{Models and Parameters}

In this work the material is described as elasto-viscoplastic, by means of the rheological model shown in Fig. 1. The outline of this particular elasto-viscoplastic model is also shown in [6]. The upper branch is purely elastic, and the associated potential is a function of the total deformation gradient $\varphi=\varphi(\mathbf{F})$. The lower (Maxwell) branch accounts for the inelastic behavior, since the inelasticity is viscoplastic, and the superscript $i$ of the inelastic deformation gradient $\mathbf{F}^{i}$ in the previous formulae is replaced in the following by $\mathrm{p}$ to represent the viscoplastic deformation gradient $\mathbf{F}^{\mathrm{p}}$. The internal variables $\mathbf{Q}$ account for the modifications of the material due to plasticity. The energy potential of the model is written as:

$$
W=\varphi(\mathbf{F})+\varphi^{\mathrm{e}}\left(\mathbf{F}^{\mathrm{e}}\right)+\varphi^{\mathrm{p}}\left(\mathbf{F}^{\mathrm{p}}, \mathbf{Q}\right)=\varphi(\mathbf{F})+\varphi^{\mathrm{e}}\left(\mathbf{F F}^{\mathrm{p}-1}\right)+\varphi^{\mathrm{p}}\left(\mathbf{F}^{\mathrm{p}}, \mathbf{Q}\right),
$$

where $\varphi(\mathbf{F})$ and $\varphi^{\mathrm{e}}\left(\mathbf{F}^{\mathrm{e}}\right)$ correspond to the elastic behavior of the springs, and $\varphi^{\mathrm{p}}\left(\mathbf{F}^{\mathrm{p}}, \mathbf{Q}\right)$ accounts for the material strain hardening. In addition, the viscous behavior is accounted for by a dissipative potential $\psi=\psi\left(\dot{\mathbf{F}}^{\mathrm{p}}, \dot{\mathbf{Q}}\right)$ which explicitly depends on the rate of change of the internal variables. In this model no dependence is assumed on the rate of the total deformation gradient $\dot{\mathbf{F}}$, i.e. $\phi=\phi(\dot{\mathbf{F}})=0$.

\subsubsection{Elastic Branch}

In this branch, the potential $\varphi(\mathbf{F})$ is split into an isochoric part, i.e., a function of the isochoric Cauchy-Green strain tensor $\hat{\mathbf{C}}$, and a volumetric part, i.e., a function of $J$, the Jacobian of $\mathbf{F}$ :

$$
\varphi(\mathbf{F})=\varphi(\hat{\mathbf{C}})+U(J) .
$$

Thus it is assumed that the whole volumetric contribution of the model is contained in $U$.

The explicit dependence on the Cauchy-Green strain holds because (as it is obvious) the energy is only related to material stretching (whilst $\mathbf{F}$ is also influenced by rigid body rotation), and thus it is possible to write:

$$
\varphi(\hat{\mathbf{C}})=\varphi\left(c_{1}, c_{2}, c_{3}\right)
$$


where $c_{1}, c_{2}, c_{3}$ are the eigenvalues of $\hat{\mathbf{C}}$.

The volumetric part is a function of the Jacobian only, and is written as:

$$
U(J)=\frac{K}{2}[\ln (J)]^{2},
$$

where $K$ is a volumetric material constant. It is evident from equation (13) that in case of an isochoric deformation $J=1$ and $U=0$.

\subsubsection{Viscoplastic Branch}

For this branch, the isochoric deformation gradient $\hat{\mathbf{F}}$ is decomposed as follows:

$$
\hat{\mathbf{F}}=\hat{\mathbf{F}}^{\mathrm{e}} \mathbf{F}^{\mathrm{p}}
$$

in which $\hat{\mathbf{F}}^{\mathrm{e}}$ is the elastic deformation gradient, corresponding to the spring, and $\mathbf{F}^{\mathrm{p}}$ is the viscoplastic deformation gradient, corresponding to the friction element, which is intrinsically isochoric $\left(\operatorname{det} \mathbf{F}^{p}=1\right)$. The viscoplastic rate of deformation tensor is assumed to coincide with the viscoplastic spatial gradient of velocity (i.e. the plastic spin is null), and so

$$
\mathbf{D}^{\mathrm{p}}=\frac{1}{2}\left(\mathbf{L}^{\mathrm{p}}+\mathbf{L}^{\mathrm{p} \mathrm{T}}\right)=\mathbf{L}^{\mathrm{p}}=\dot{\mathbf{F}}^{\mathrm{p}} \mathbf{F}^{\mathrm{p}-1} .
$$

From equation (15) the flow rule follows:

$$
\dot{\mathbf{F}}^{\mathrm{p}}=\mathbf{D}^{\mathrm{p}} \mathbf{F}^{\mathrm{p}} .
$$

The viscoplastic rate of deformation tensor is expressed by means of a spectral decomposition, with the additional assumptions of a von Mises-type plasticity model:

$$
\begin{gathered}
\mathbf{D}^{\mathrm{p}}=\dot{q} \sum_{j=1}^{3} q_{j} \mathbf{m}_{j} \otimes \mathbf{m}_{j}=\dot{q} \sum_{j=1}^{3} q_{j} \mathbf{M}_{j}, \\
q_{j} \in K_{Q}=\left\{p_{j} \in \Re, \sum_{j=1}^{3} p_{j}=0 ; \sum_{j=1}^{3} p_{j}^{2}=3 / 2\right\}, \\
\mathbf{M}_{j} \in K_{M}=\left\{\mathbf{N}_{j} \in \operatorname{Sym} ; \mathbf{N}_{j}: \mathbf{N}_{j}=1, \mathbf{N}_{i}: \mathbf{N}_{j}=0, i \neq j\right\},
\end{gathered}
$$

where $\dot{q}$ is the rate of accumulated plastic deformation, $q_{j}$ are linear combination coefficients, $\mathbf{m}_{j}$ and $\mathbf{M}_{j}$ are, respectively, the eigenvalues and the eigenprojections of $\mathbf{D}^{\mathrm{p}}$. The conditions of isochoric deformation and the radius of the von Mises yield surface (set of scalars $K_{Q}$ ) are enforced by equation (18), while equation (19) enforces the eigenprojections to be symmetric orthogonal (set of second-order tensors $K_{M}$ ).

The viscoplastic deformations are incrementally updated by means of an exponential mapping. The increments of viscoplastic deformation gradient and viscoplastic Cauchy-Green strain are respectively:

$$
\Delta \mathbf{F}^{\mathrm{p}}=\mathbf{F}_{n+1}^{\mathrm{p}} \mathbf{F}_{n}^{\mathrm{p}-1}=\Delta \mathbf{R} \exp \left[\Delta t \mathbf{D}^{\mathrm{p}}\right],
$$




$$
\Delta \mathbf{C}^{\mathrm{p}}=\left(\Delta \mathbf{F}^{\mathrm{p}}\right)^{\mathrm{T}} \Delta \mathbf{F}^{\mathrm{p}}=\mathbf{F}_{n}^{\mathrm{p}-\mathrm{T}} \mathbf{C}_{n+1}^{\mathrm{p}} \mathbf{F}_{n}^{\mathrm{p}-1}=\exp \left[\Delta t \mathbf{D}^{\mathrm{p}}\right]^{2},
$$

where the rotation increment $\Delta \mathbf{R}$ comes from the polar decomposition of $\Delta \mathbf{F}^{\mathrm{p}}$. From equation (21) and considering equation (17) it follows that

$$
\frac{1}{2} \ln \left(\Delta \mathbf{C}^{\mathrm{p}}\right)=\Delta t \mathbf{D}^{\mathrm{p}}=\Delta t \dot{q} \sum_{j=1}^{3} q_{j} \mathbf{M}_{j}=\Delta q \sum_{j=1}^{3} q_{j} \mathbf{M}_{j},
$$

where $\Delta q=\Delta t \dot{q}$. Substituting equation (22) in equation (20) it is found that

$$
\mathbf{F}_{n+1}^{p}=\exp \left[\Delta q \sum_{j=1}^{3} q_{j} \mathbf{M}_{j}\right] \mathbf{F}_{n}^{\mathrm{p}}
$$

Here the rotation $\Delta \mathbf{R}$ appearing in equation (20) has been disregarded since the constitutive expressions depend on the Cauchy-Green tensor in which the rotation terms are eliminated. The potentials of the viscoplastic branch can now be defined by means of the internal variables $q, q_{j}$ and $\mathbf{M}_{j}$ only.

The elastic potential of the branch is written as a function of the eigenvalues of $\hat{\mathbf{C}}^{\mathrm{e}}$, similarly to the case of equation (12):

$$
\varphi^{\mathrm{e}}\left(\hat{\mathbf{C}}^{\mathrm{e}}\right)=\varphi^{\mathrm{e}}\left(c_{1}^{\mathrm{e}}, c_{2}^{\mathrm{e}}, c_{3}^{\mathrm{e}}\right)
$$

The plastic potential $\varphi^{\mathrm{p}}$ accounts for the strain hardening of the material. In case of isotropic hardening the potential is defined as

$$
\varphi^{\mathrm{p}}=\varphi^{\mathrm{p}}(q), \quad \text { where } q=\int_{0}^{t} \dot{q} \mathrm{~d} t
$$

In incremental terms the accumulated plastic deformation can be written as:

$$
q_{n+1}=q_{n}+\Delta t \dot{q}=q_{n}+\Delta q .
$$

The dissipative viscoplastic potential $\psi$ depends on $\mathbf{D}^{\mathrm{p}}$ and, under the adopted hypotheses, on $\dot{q}$ only:

$$
\psi\left(\mathbf{D}^{\mathrm{p}}\right)=\psi\left(\frac{\Delta q}{\Delta t}\right)=\psi(\dot{q})= \begin{cases}\bar{\psi}(\dot{q}), & \text { if } \dot{q} \geqslant 0, \\ +\infty, & \text { if } \dot{q}<0,\end{cases}
$$

where in the first case $\bar{\psi}$ is a convenient formula for the potential, whilst the second case has the meaning of a penalization that constraints the rate $\dot{q}$ to be non-negative.

\subsection{Adopted Models}

Within this work the following models have been used in the potentials.

\subsubsection{Elastic Potential}

Two different models have been tested to account for the elastic behavior. The first is the Hencky model

$$
\varphi^{\mathrm{e}}=\mu^{\mathrm{e}} \sum_{j=1}^{3}\left(\varepsilon_{j}^{\mathrm{e}}\right)^{2}
$$


in which $\mu^{\mathrm{e}}$ is a material constant, and $\varepsilon_{j}^{\mathrm{e}}$ the $j$ th component of elastic logarithmic strain. Alternatively, also Ogden-type models have been tested:

$$
\varphi^{\mathrm{e}}=\sum_{j=1}^{3} \sum_{k=1}^{N^{\mathrm{e}}} \frac{\mu_{k}^{\mathrm{e}}}{\alpha_{k}^{\mathrm{e}}}\left(\left(\exp \left(\varepsilon_{j}^{\mathrm{e}}\right)\right)^{\alpha_{k}^{\mathrm{e}}}-1\right)
$$

where $\mu_{k}^{\mathrm{e}}$ and $\alpha_{k}^{\mathrm{e}}$ are material constants, and $N^{\mathrm{e}}$ is the number of terms included.

\subsubsection{Plastic Potential}

A general model has been tested in the form

$$
\varphi^{\mathrm{p}}=\Sigma_{0} q+\frac{1}{2} H q^{2}+\mu\left(q+\frac{1}{\alpha} \exp (-\alpha q)\right)+\sum_{k=1}^{N^{\mathrm{p}}} \frac{\mu_{k}^{\mathrm{p}}}{\alpha_{k}^{\mathrm{p}}+1}(q)^{\alpha_{k}^{\mathrm{p}}+1},
$$

in which $\Sigma_{0}$ is the yield stress and $H$ is the plastic modulus (linear hardening), $\mu$ and $\alpha$ are material constants of the exponential law (saturation), and $\mu_{k}^{\mathrm{p}}$ and $\alpha_{k}^{\mathrm{p}}$ are material constants of the power law that includes $N^{\mathrm{p}}$ terms. According to the coefficients included, equation (30) can account for many material models adopted in plasticity.

\subsubsection{Viscous Potential}

The Perzyna [5] potential

$$
\psi(\dot{q})= \begin{cases}\frac{m Y_{0} \dot{q}_{0}}{m+1}\left(\frac{\dot{q}}{\dot{q}_{0}}\right)^{(m+1) / m}, & \text { if } \dot{q} \geqslant 0, \\ +\infty, & \text { if } \dot{q}<0,\end{cases}
$$

has been adopted where $m$ and $Y_{0}$ are material constants, $\dot{q}_{0}$ is a reference rate. In this work it is assumed that $Y_{0}$ coincides with the yield stress $\Sigma_{0}$.

\subsection{Minimization at Each Increment}

It is assumed that the potential $\varphi(\hat{\mathbf{F}})$ is null, thus only the volumetric contribution $U(J)$ can be present in the elastic branch. The minimization problem (8) can be rewritten as

$$
\begin{aligned}
\Psi\left(\mathbf{F}_{n+1}\right) & =\Psi\left(\mathbf{C}_{n+1}\right) \\
& =\Delta U\left(J_{n+1}\right)+\min _{\Delta q, \mathbf{M}_{j}, q_{j}}\left\{\Delta \varphi^{\mathrm{e}}\left(\hat{\mathbf{C}}_{n+1}^{\mathrm{e}}\right)+\Delta \varphi^{\mathrm{p}}\left(q_{n+1}\right)+\Delta t \psi\left(\frac{\Delta q}{\Delta t}\right)\right\},
\end{aligned}
$$

where:

$$
\begin{gathered}
\Delta U\left(J_{n+1}\right)=U\left(J_{n+1}\right)-U\left(J_{n}\right), \\
\Delta \varphi^{\mathrm{e}}\left(\hat{\mathbf{C}}_{n+1}^{\mathrm{e}}\right)=\varphi^{\mathrm{e}}\left(\hat{\mathbf{C}}_{n+1}^{\mathrm{e}}\right)-\varphi^{\mathrm{e}}\left(\hat{\mathbf{C}}_{n}^{\mathrm{e}}\right), \\
\Delta \varphi^{\mathrm{p}}\left(q_{n+1}\right)=\varphi^{\mathrm{p}}\left(q_{n+1}\right)-\varphi^{\mathrm{p}}\left(q_{n}\right) .
\end{gathered}
$$


During each increment, the internal variables $q_{j}$ and $\mathbf{M}_{j}$ must satisfy the conditions (18) and (19), respectively, and $\Delta q \geqslant 0$.

Following a procedure similar to that shown in [3], it is possible to show that the minimization with respect to $\mathbf{M}_{j}$ implies co-linearity of $\hat{\mathbf{C}}_{n+1}^{\mathrm{e}}, \hat{\mathbf{C}}^{\text {pr }}=$ $\mathbf{F}_{n}^{\mathrm{p}-\mathrm{T}} \hat{\mathbf{C}}_{n+1} \mathbf{F}_{n}^{\mathrm{p}-1}$ and $\mathbf{D}^{\mathrm{p}}$. The minimum conditions with respect to $\Delta q, q_{j}$ give the system of simultaneous non-linear equations:

$$
\begin{gathered}
-\frac{\partial \Delta \varphi^{\mathrm{e}}}{\partial \varepsilon_{i}^{\mathrm{e}}} \Delta q+\beta_{1}+2 \beta_{2} q_{i}=0, \quad i=1,2,3, \\
-\sum_{j=1}^{3} \frac{\partial \Delta \varphi^{\mathrm{e}}}{\partial \varepsilon_{i}^{\mathrm{e}}} q_{j}+\frac{\partial \Delta \varphi^{\mathrm{p}}}{\partial \Delta q}+\frac{\partial \psi}{\partial \dot{q}}=0, \\
\sum_{j=1}^{3} q_{j}=0, \\
\sum_{j=1}^{3} q_{j}^{2}-\frac{3}{2}=0,
\end{gathered}
$$

in which, besides the already mentioned variables $\Delta q, q_{1}, q_{2}, q_{3}$, also appear the Lagrange multipliers $\beta_{1}$ and $\beta_{2}$ which enforce the conditions of isochoric deformation and the radius of the von Mises yield surface. The system of equations is solved numerically.

The particular case of uniaxial stress is treated without loss of generality. After minimization, the Piola-Kirchhof stress is evaluated as

$$
\begin{aligned}
\mathbf{P}_{n+1} & =2 \mathbf{F}_{n+1} \frac{\partial \Psi\left(\mathbf{C}_{n+1}\right)}{\partial \mathbf{C}_{n+1}} \\
& =\mathbf{F}_{n+1}\left[2 J_{n+1}^{-2 / 3} \operatorname{DEV}\left(\frac{\partial \varphi}{\partial \hat{\mathbf{C}}_{n+1}}+\frac{\partial \varphi^{\mathrm{e}}}{\partial \hat{\mathbf{C}}_{n+1}}+\Delta t \frac{\partial \phi}{\partial \hat{\mathbf{C}}_{n+1}}\right)+\frac{\partial U}{\partial J_{n+1}} J_{n+1} \mathbf{C}_{n+1}^{-1}\right],
\end{aligned}
$$

where $\operatorname{DEV}(\mathbf{A})=\mathbf{A}-\frac{1}{3}(\mathbf{A}: \mathbf{C}) \mathbf{C}^{-1}$. Assuming isochoric behavior $(J=1)$ the deformation gradient $\mathbf{F}$ and the Cauchy-Green strain $\mathbf{C}$ tensors become:

$$
\mathbf{F}=\hat{\mathbf{F}}=\left[\begin{array}{ccc}
\lambda & 0 & 0 \\
0 & 1 / \sqrt{\lambda} & 0 \\
0 & 0 & 1 / \sqrt{\lambda}
\end{array}\right]
$$

and

$$
\mathbf{C}=\hat{\mathbf{C}}=\mathbf{F}^{2},
$$

where $\lambda$ is the stretch in the uniaxial loading direction. 
Denoting $\sigma=\sigma_{11}$, the only non-zero component of the Cauchy stress, the volumetric incremental relationship becomes

$$
p_{n+1}=\frac{\partial U}{\partial J_{n+1}}=\frac{\sigma}{3} .
$$

Substituting expressions (41), (42) and (43) in (40), and recalling the relationship $\sigma=J^{-1} \mathbf{P F}^{\mathrm{T}}$ between Cauchy and Piola-Kirchhoff stress tensors, it is found that

$$
\sigma_{n+1}=\mathbf{C}_{n+1}\left[2 \operatorname{DEV}\left(\frac{\partial \varphi}{\partial \hat{\mathbf{C}}_{n+1}}+\frac{\partial \varphi^{\mathrm{e}}}{\partial \hat{\mathbf{C}}_{n+1}}+\Delta t \frac{\partial \phi}{\partial \hat{\mathbf{C}}_{n+1}}\right)+p_{n+1} \mathbf{C}_{n+1}^{-1}\right]
$$

\section{Experimental}

\subsection{Test Configuration}

The experimental tests, described in more detail in [7], were designed to investigate the influence of the strain rate on the material response over a wide range, from $1 \times$ $10^{-3} \mathrm{~s}^{-1}$ to $3 \times 10^{3} \mathrm{~s}^{-1}$. The stress-strain curves were measured from specimens made entirely of the adhesive, to avoid the influence of the adherends and joint geometry on the results.

Due to the very large range of strain rates considered, two different test facilities were needed: the tests at strain rates lower than $20 \mathrm{~s}^{-1}$ were carried out on a universal servo-hydraulic testing machine, while the tests at higher strain rates, up to $3.0 \times 10^{3} \mathrm{~s}^{-1}$, were carried out on a Split Hopkinson Pressure Bar (SHPB). Traditionally, the use of the SHPB has been typical for compression tests on metals [8], but in recent years several studies [9-12] have considered other kinds of materials like polymers, foams, ceramics, etc. and have also tested under different stress states (tension, torsion, bending) by means of special set-ups. However, studies specifically concerning adhesives are relatively rare [13, 14].

The standard SHPB configuration performs dynamic compression tests, usually on cylindrical specimens. Figure 2 shows the simplified sketch of a compression SHPB. The test starts when the impact of the striker bar against the input bar generates a compression pulse. The pulse propagates along the input bar, then it is partially reflected and partially transmitted through the specimen and the output bar. By measuring the reflected pulse on the input bar and the transmitted pulse on

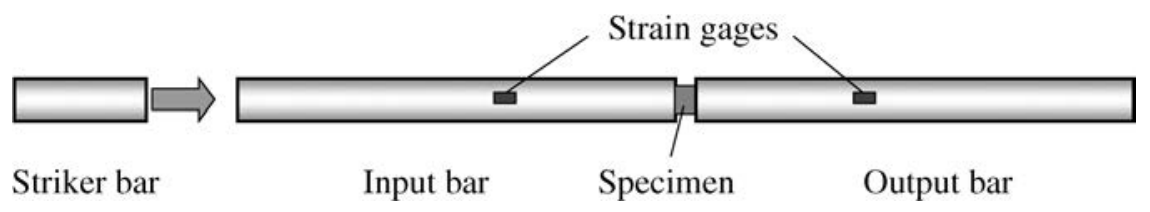

Figure 2. SHPB compression test configuration. 
the output bar it is possible to reconstruct the dynamic stress-strain curve of the tested material by means of the following equations [8]:

$$
\begin{gathered}
\sigma_{\text {specimen }}(t)=E_{0} \frac{A_{0}}{A} \varepsilon_{\text {transmitted }}(t), \\
\varepsilon_{\text {specimen }}(t)=-\frac{2 c_{0}}{L} \int \varepsilon_{\text {reflected }}(t) \cdot \mathrm{d} t, \\
\dot{\varepsilon}_{\text {specimen }}(t)=-\frac{2 c_{0}}{L} \varepsilon_{\text {reflected }}(t),
\end{gathered}
$$

where $A$ and $L$ are, respectively, the cross-section and length of the specimen; $A_{0}, c_{0}$ and $E_{0}$ are cross-section, wave velocity and elastic modulus of the bar, respectively.

Among the cases of structural adhesives and curing methods tested in [7], the results chosen for this work are those on the bi-component, cold cured Loctite Hysol 9466 epoxy (Henkel, Düsseldorf, Germany). All specimens were made by injecting the adhesive into Teflon ${ }^{\circledR}$ moulds and curing them at room temperature for $24 \mathrm{~h}$. After removal they were aged for one week before testing. The specimens for static and dynamic compression tests were cylinders with a diameter of $10 \mathrm{~mm}$. For the tests carried out on the hydraulic machine (on which the crosshead speed can be adjusted), the adopted length values were $10 \mathrm{~mm}$ for strain rates up to $0.5 \times 10^{1} \mathrm{~s}^{-1}$ and $4 \mathrm{~mm}$ for higher strain rates. The length values adopted to obtain the desired nominal (in the sense that the actual strain rate depends also on the intensity of the impact and can be assessed only after the test) strain rate on the SHPB varied from $4 \mathrm{~mm}\left(3.0 \times 10^{3} \mathrm{~s}^{-1}\right)$ to $10 \mathrm{~mm}\left(1.5 \times 10^{3} \mathrm{~s}^{-1}\right)$.

In [7] are reported also cases of tensile tests carried out on specimens with threaded ends. To test them at high strain rates, a modified version of the SHPB was used, in which the tensile pulse is created by means of a reflection system [12]. Due to the lower precision of this system, the results obtained were not suitable for the type of processing carried out in the present work, thus only results from compression tests are considered here. Moreover, the failures in these tension tests are - unlike the case of compression — substantially brittle and thus give little information about the inelastic behavior of the adhesive.

\subsection{Results}

Figure 3 shows the results of the compression tests for the cold-cured samples (two replicates for each case). The progressive growth of the curves under increasing strain rate can be noticed and the dynamic stress-strain curves present approximately the same shape as the static ones. A generic curve contains: an elastic initial part (linear with good approximation); a rounded knee; and a wide plateau, initially softening (at high strain rate) then progressively hardening. The shorter extension of the curves corresponding to nominal strain rate from $1.2 \times 10^{3}$ to $2.0 \times 10^{3} \mathrm{~s}^{-1}$ is due to the fact that, under the same displacement applied by the bar to the specimen end, the longer the specimen, the lower the total strain. The relevant corresponding 


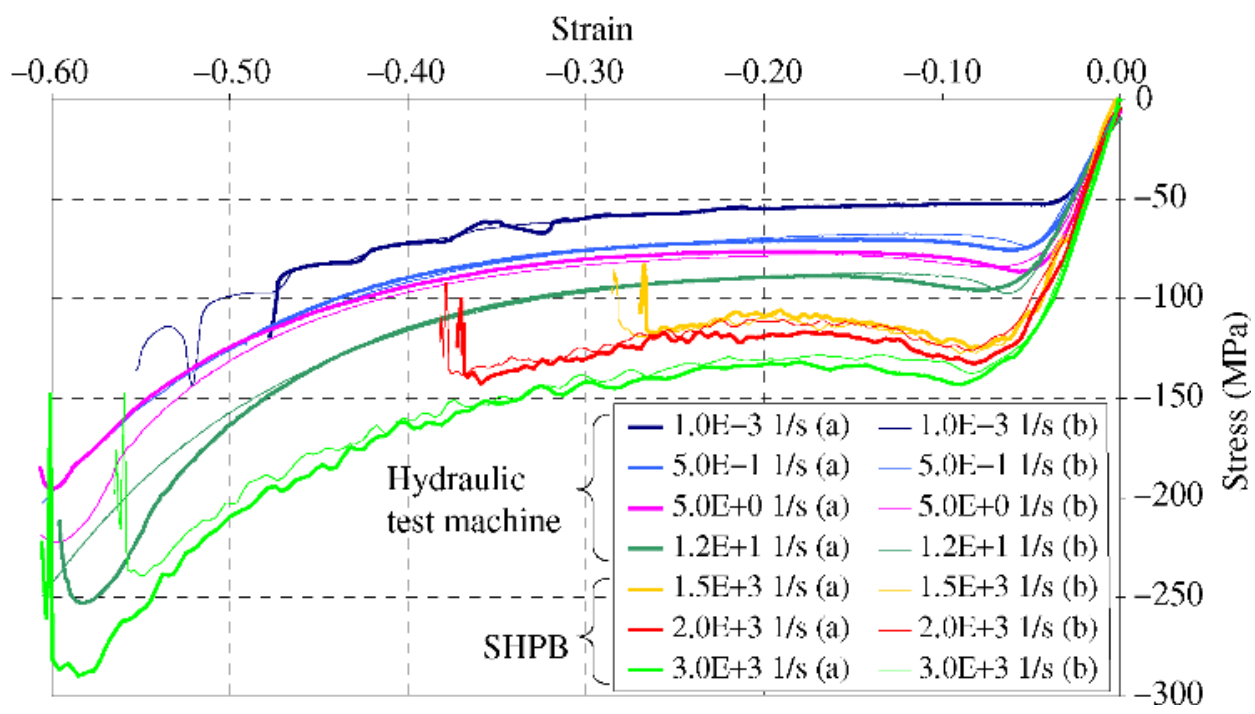

Figure 3. Experimental compression test results on the Hysol 9466 at different strain rates (a and b denote the two replicates).

Table 1.

Nominal strain rate, actual mean strain rate, elastic limit, $2 \%$ proof stress (for each replicate the values are separated by "," in the box)

\begin{tabular}{lllll}
\hline & $\dot{\varepsilon}_{\text {nominal }}\left(\mathrm{s}^{-1}\right)$ & $\dot{\varepsilon}_{\text {actual,mean }}\left(\mathrm{s}^{-1}\right)$ & $E(\mathrm{GPa})$ & $R_{\mathrm{p} 0.2}(\mathrm{MPa})$ \\
\hline Static tests & $1.0 \times 10^{-3}$ & $1.00 \times 10^{-3}, 1.00 \times 10^{-3}$ & $1.98,2.20$ & $-47,-47$ \\
& $1.0 \times 10^{-2}$ & $1.10 \times 10^{-2}, 1.00 \times 10^{-2}$ & $2.00,1.99$ & $-52,-53$ \\
Dynamic tests & $1.0 \times 10^{-1}$ & $1.15 \times 10^{-1}, 1.13 \times 10^{-1}$ & $1.92,1.93$ & $-60,-61$ \\
$\quad 5.0 \times 10^{-1}$ & $5.23 \times 10^{-1}, 5.11 \times 10^{-1}$ & $1.85,1.90$ & $-74,-75$ \\
Hydraulic machine & $5.0 \times 10^{0}$ & $4.83 \times 10^{0}, 4.98 \times 10^{0}$ & $2.01,2.25$ & $-86,-86$ \\
& $1.2 \times 10^{1}$ & $1.21 \times 10^{1}, 1.21 \times 10^{1}$ & $1.99,1.95$ & $-93,-95$ \\
Dynamic tests & $1.5 \times 10^{3}$ & $1.49 \times 10^{3}, 1.48 \times 10^{3}$ & $(2.17,2.24)$ & $-126,-122$ \\
SHPB & $2.0 \times 10^{3}$ & $2.01 \times 10^{3}, 2.00 \times 10^{3}$ & $(2.30,2.09)$ & $-131,-126$ \\
& $3.0 \times 10^{3}$ & $3.04 \times 10^{3}, 2.99 \times 10^{3}$ & $(2.44,2.30)$ & $-143,-142$ \\
\hline
\end{tabular}

Data in parentheses are affected by measurement inaccuracy but are reported for completeness only.

numerical data are reported in Table 1. As expected, the values of elastic modulus at high strain rate are in general slightly higher than those obtained statically or at low strain rate. Much more relevant is the increase in absolute value of the elastic limit with the strain rate, that changes from about $-47 \mathrm{MPa}$ in static condition to about $-143 \mathrm{MPa}$ at $3.0 \times 10^{3} \mathrm{~s}^{-1}$. 


\section{Identification of the Parameters}

The uniaxial case has been implemented in the MATLAB ${ }^{\circledR}$ code (The MathWorks Inc., Natick, MA, USA), with the aim of building a tool able to determine the values of the different parameters that appear in the models by reproducing the experimental results. The advantage of doing so is that the computation time is much shorter than it would be required using an actual FEM code; moreover, no additional problem related to discretization (meshing, element formulation) is introduced.

The identification of the parameters is based on a least squares algorithm that minimizes the difference between model response and measurements. The experimental curves are the average of the two replicates. For the elastic potentials both the Hencky and $\operatorname{Ogden}\left(N^{\mathrm{e}}=1,2,3\right)$ models were tried. The plastic potential cases have been tested with $H$ forced to be zero or left unconstrained, and with the number of terms in the power law $N^{\mathrm{p}}=1,2,3$. The viscous potential is in all cases that of Perzyna. Table 2 gives a complete list of the tested combinations, for a total of 24 cases. The number of parameters simultaneously identified varies from 8 (elastic potential Hencky, case 1) to 18 (elastic potential Ogden $N^{\mathrm{e}}=3$, case 6). The computation time is of the order of some hours per case on a 64 bit PC. For the sake of brevity only a small selection of the results from the 24 cases is reported here, in terms of the corresponding strain-stress diagrams and values of the parameters.

Regarding the role of the elastic potential, the use of the Ogden $v s$. the simpler Hencky model was tested to account for the possible non-linear elasticity. It was found that for this material the Ogden model did not offer any advantages. This is probably caused by the fact that the " $\mathrm{S}$ " shape of the curves is due to plasticity and not to hyperelasticity: the elastic behavior is substantially linear and, therefore, the Hencky model is already adequate.

Regarding the plastic potential, the tests have tried to identify the contributions of the different terms, in particular that of linear strain hardening $(H)$ and that of the

Table 2.

List of the 24 tested combinations of models for the elastic and plastic potentials

\begin{tabular}{|c|c|c|c|c|c|c|}
\hline \multirow{3}{*}{$\begin{array}{l}\text { Elastic potential } \\
\text { Hencky }\end{array}$} & \multicolumn{6}{|c|}{ Plastic potential } \\
\hline & \multicolumn{3}{|l|}{$H=0$} & \multicolumn{3}{|c|}{$H$ unconstrained } \\
\hline & $N^{\mathrm{p}}=1$ & $N^{\mathrm{p}}=2$ & $N^{\mathrm{p}}=3$ & $N^{\mathrm{p}}=1$ & $N^{\mathrm{p}}=2$ & $N^{\mathrm{p}}=3$ \\
\hline Case & 1 & 2 & 3 & 4 & 5 & 6 \\
\hline $\operatorname{Ogden} N^{\mathrm{e}}=1$ & $N^{\mathrm{p}}=1$ & $N^{\mathrm{p}}=2$ & $N^{\mathrm{p}}=3$ & $N^{\mathrm{p}}=1$ & $N^{\mathrm{p}}=2$ & $N^{\mathrm{p}}=3$ \\
\hline Case & 1 & 2 & 3 & 4 & 5 & 6 \\
\hline $\operatorname{Ogden} N^{\mathrm{e}}=2$ & $N^{\mathrm{p}}=1$ & $N^{\mathrm{p}}=2$ & $N^{\mathrm{p}}=3$ & $N^{\mathrm{p}}=1$ & $N^{\mathrm{p}}=2$ & $N^{\mathrm{p}}=3$ \\
\hline Case & 1 & 2 & 3 & 4 & 5 & 6 \\
\hline $\operatorname{Ogden} N^{\mathrm{e}}=3$ & $N^{\mathrm{p}}=1$ & $N^{\mathrm{p}}=2$ & $N^{\mathrm{p}}=3$ & $N^{\mathrm{p}}=1$ & $N^{\mathrm{p}}=2$ & $N^{\mathrm{p}}=3$ \\
\hline Case & 1 & 2 & 3 & 4 & 5 & 6 \\
\hline
\end{tabular}

Viscous potential: Perzyna in all cases. 
power law. The yield $\Sigma_{0}$ and the exponential part of the potential (more adequate to describe the knee) have always been included. In general, all cases have proven to perform satisfactorily, in the sense that the experimental curves are reproduced, even when a limited number of parameters are used. The major improvement that can be achieved, by increasing the complexity of the model, is obtained when going from cases 1, 2 and 4 (quite similar to each other) to cases 3, 5 and 6 (also similar to each other) that are able to reproduce - at least the trend - the softening. The general conclusion is that a model of reasonable complexity can reproduce the desired behavior; for instance, the case with linear strain hardening and $N^{\mathrm{p}}=1$ already shows post-yield softening.

As an example, the cases Hencky \# $1\left(H=0, N^{\mathrm{p}}=1\right)$, Hencky \# 5 ( $H$ unconstrained, $N^{\mathrm{p}}=2$ ), and the most complex Ogden $N^{\mathrm{e}}=3 \# 6$ ( $H$ unconstrained, $N^{\mathrm{p}}=3$ ) are plotted in Figs 4, 5 and 6, respectively. Table 3 lists the corresponding parameters. The main difference among them is that the simplest case of Fig. 4 does not describe the stress peak and the subsequent slight softening, which are already (even if not completely) reproduced by the case in Fig. 5. It can be noticed from Fig. 6 that no significant improvement is added by the most complex case.

Regarding the viscous potential, it is interesting to notice how the implemented model accounts for the strain rate dependence by reproducing quite well the experimental curves, in a strain rate range that spans over six orders of magnitude $\left(10^{-3}-10^{3} \mathrm{~s}^{-1}\right)$. This justifies the choice of the potential adopted, although the Perzyna model had been initially proposed (and usually applied) for materials with linear strain hardening. The $m$ coefficient in (31) remains approximately in the range $8-9$ in all cases. Conversely, the reference rate $\dot{q}_{0}$ interacts with the other

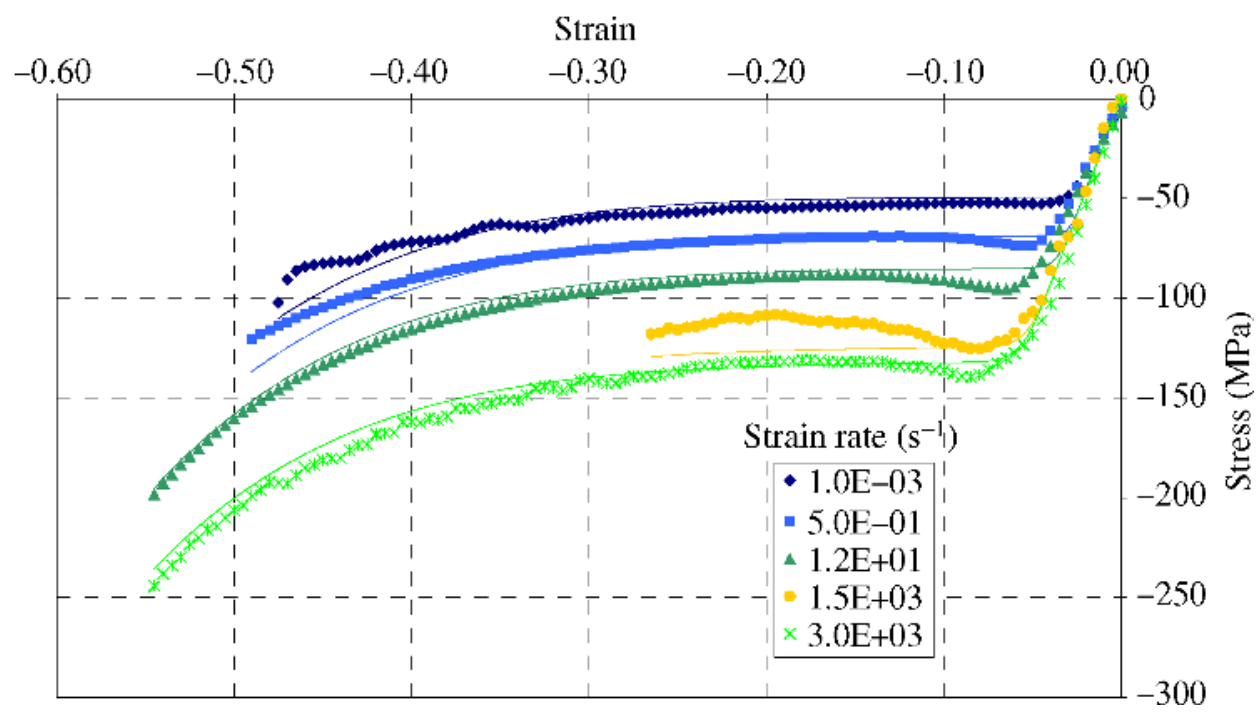

Figure 4. Experimental (symbols) and simulated (lines) stress-strain curves at different strain rates; model Hencky \# 1. 


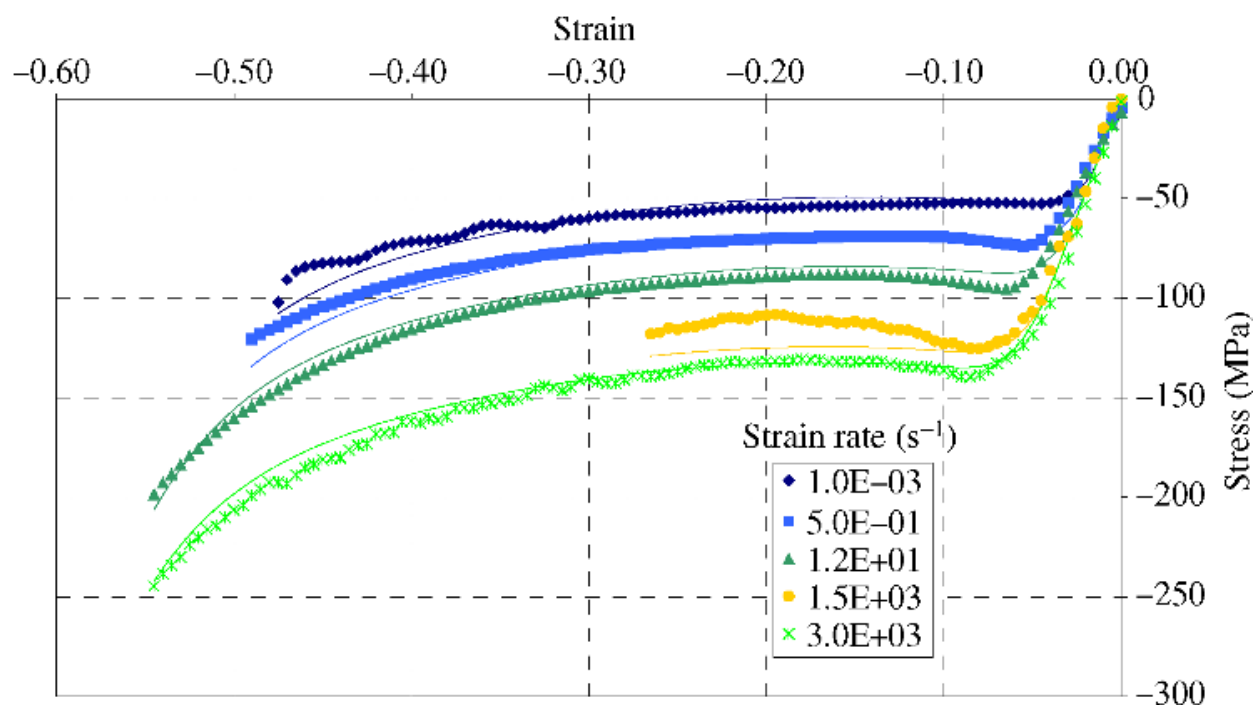

Figure 5. Experimental (symbols) and simulated (lines) stress-strain curves at different strain rates; model Hencky \# 5.

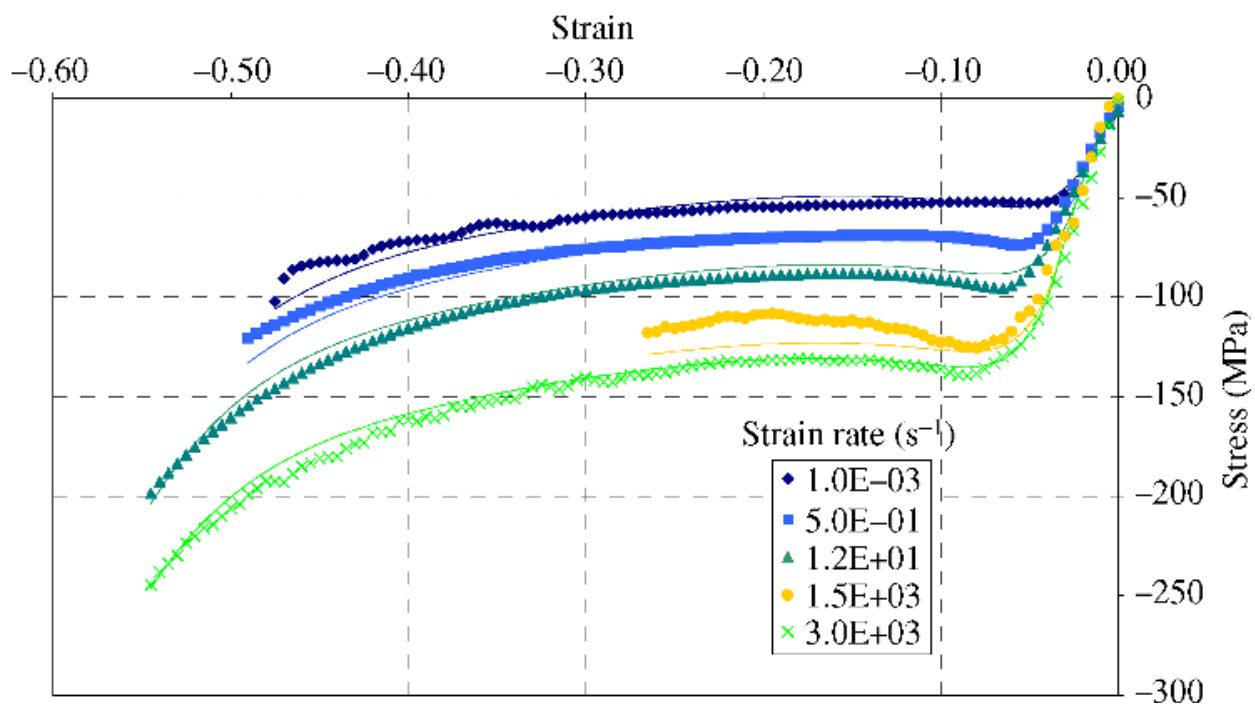

Figure 6. Experimental (symbols) and simulated (lines) stress-strain curves at different strain rates; model Ogden $N^{\mathrm{e}}=3 \# 6$.

parameters of the simulation and is more subjected to changes from case to case. Considering the graphs presented in Figs 4, 5 and 6, the only objection that can be raised is that if one extrapolates the curves of the model towards higher strain, they all tend to superpose each other. This behavior, physically not plausible, is due to 
Table 3.

Model parameters for the cases Hencky \# 1, Hencky \# 5, Ogden $N^{\mathrm{e}}=3$ \# 6

\begin{tabular}{llll}
\hline & Hencky \# 1 & Hencky \# 5 & Ogden $N^{\mathrm{e}}=3$ \# 6 \\
\hline$\mu_{1}^{\mathrm{e}}, \mu^{\mathrm{e}}$ & 759.17 & 775.76 & 15171 \\
$\mu_{2}^{\mathrm{e}}$ & - & - & 988.17 \\
$\mu_{3}^{\mathrm{e}}$ & - & - & -1508.2 \\
$\alpha_{1}^{\mathrm{e}}$ & - & - & 0.82038 \\
$\alpha_{2}^{\mathrm{e}}$ & - & - & 0.85850 \\
$\alpha_{3}^{\mathrm{e}}$ & - & - & 8.1714 \\
$\Sigma_{0}$ & 24.994 & 22.525 & 15.507 \\
$H$ & - & -202.74 & -342.23 \\
$\mu$ & 5.0436 & 17.970 & 32.330 \\
$\alpha$ & 80798 & 115.01 & 75.979 \\
$\alpha_{1}^{\mathrm{p}}$ & 3.5871 & 10.069 & 5.6594 \\
$\alpha_{2}^{\mathrm{p}}$ & - & 1.4307 & 1.5239 \\
$\alpha_{3}^{\mathrm{p}}$ & - & - & 3.2143 \\
$\mu_{1}^{\mathrm{p}}$ & 381.44 & 2002.4 & 942.59 \\
$\mu_{2}^{\mathrm{p}}$ & - & 332.53 & 660.63 \\
$\mu_{3}^{\mathrm{p}}$ & - & - & -575.49 \\
$\dot{q}_{0}$ & $1.0628 \times 10^{-2}$ & $1.2147 \times 10^{-2}$ & $5.0476 \times 10^{-4}$ \\
$m$ & 8.9796 & 8.4443 & 8.5219 \\
\hline
\end{tabular}

the mathematical structure of the potential. Nevertheless, in the strain range tested the model response is correct.

\section{Conclusions}

The variational formulation of the elasto-viscoplastic material model has been applied to the case of a structural bi-component epoxy adhesive, with the aim of reproducing its behavior under compression at different test velocities. The work has been carried out within a variational framework previously developed, that makes it possible to tackle the problem at general level, accommodating different models according to the specific potentials implemented. The natural use of this formulation is the implementation in non-linear finite element codes. However, in the present work it has been utilized analytically, due to the uni-axiality of the problem.

The main aspects which have been considered in this work are the choice of the potentials and the evaluation of the capability for reproducing the experiments. As far as elasticity is concerned, since this adhesive is stiff and linear elastic, a Hencky potential shows to be adequate. Regarding plasticity, the adopted potential — which combines the typical behaviors (linear hardening, saturation, power law) - is also 
able to reproduce material softening with a limited number of parameters. Finally, what is perhaps more remarkable is the capability of the adopted Perzyna potential to fit the curves at different strain rates, from quasi-static conditions to $103 \mathrm{~s}^{-1}$.

\section{Acknowledgements}

Jakson Manfredini Vassoler is presently enrolled in his PhD course at the Politecnico di Torino under the sponsorship of the ALPIP (America Latina Plemonte Politecnico) interchange program.

Eduardo Fancello also thanks the Brazilian organization CNPq that provided partial financial support for this research.

Laurent Stainier is a Research Associate at the Belgian "Fonds de la Recherche Scientifique (F.R.S.-FNRS)".

\section{References}

1. M. Ortiz and L. Stainier, Computer Meth. Appl. Mech. Eng. 171, 419 (1999).

2. E. H. Lee, J. Appl. Mech. 36, 1 (1969).

3. E. Fancello, J. P. Ponthot and L. Stainier, Int. J. Numer. Meth. Eng. 65, 1831 (2006).

4. M. Ortiz and E. A. Repetto, J. Mech. Phys. Solids 47, 397 (1999).

5. P. Perzyna, Int. J. Solids Struct. 22, 797 (1986).

6. E. Fancello, J. M. Vassoler and L. Stainier, in: Proceedings of the IX International Conference on Computational Plasticity, Barcelona, Spain, p. 273 (2007).

7. L. Goglio, M. Peroni and M. Rossetto, Key Eng. Mater. 347, 671 (2007).

8. H. Kolsky, Stress Waves in Solids. Dover Publications, New York, NY (1963).

9. J. P. Hou, C. Ruiz and A. Trojanowski, Mater. Sci. Eng. A 283, 181 (2000).

10. L. Ninan, J. Tsai and C. T. Sun, Int. J. Impact Eng. 25, 291 (2001).

11. W. Chen, F. Lu and N. Winfree, Exp. Mech. 42, 65 (2002).

12. W. Chen, F. Lu and M. Cheng, Polymer Test. 21, 113 (2002).

13. M. A. Martínez, I. S. Chocron, J. Rodríguez, V. S. Gálvez and L. A. Sastre, Int. J. Adhesion Adhesives 18, 375 (1998).

14. T. Yokoyama, J. Strain Anal. 38, 233 (2003). 